\title{
The relation between mirror movements and non-use of the affected hand in children with unilateral cerebral palsy
}

\author{
INGAR M ZIELINSKI ${ }^{1}$ | DIDO GREEN ${ }^{2}$ | JULIAN RUDISCH ${ }^{2}$ | MARIJTJE L A JONGSMA ${ }^{1}$ | PAULINE B M AARTS ${ }^{3}$ | \\ BERT STEENBERGEN ${ }^{1,4}$
}

1 Behavioural Science Institute, Nijmegen, the Netherlands. 2 Department of Sport and Health Science, Oxford Brookes University, Oxford, UK. 3 Department of Pediatric Rehabilitation, Sint Maartenskliniek, Nijmegen, the Netherlands. 4 School of Psychology, Australian Catholic University, Melbourne, Vic., Australia.

Correspondence to Ingar M Zielinkski at Postbus 9104, 6500 HE Nijmegen, the Netherlands. E-mail: i.zielinski@pwo.ru.nl

This article is commented on by Standt on pages 114-115 of this issue.

\section{PUBLICATION DATA}

Accepted for publication 8th June 2016.

Published online 16th July 2016.

\section{ABBREVIATIONS}

$\mathrm{CCC}_{\max } \quad$ Maximum cross-correlation coefficient

LAH Less-affected hand

MACS Manual Ability Classification System

$\mathrm{MM}_{\text {strength }}$ Strength of mirror movements
AIM In children with unilateral cerebral palsy (CP), it is widely believed that mirror movements contribute to non-use of the affected hand despite preserved capacity, a phenomenon referred to as developmental disregard. We aimed to test whether mirror movements are related to developmental disregard, and to clarify the relation between mirror movements and bimanual function.

METHOD A repetitive squeezing task simultaneously measuring both hands' grip-forces was developed to assess mirror movements by using maximum cross-correlation coefficient $\left(\mathrm{CCC}_{\max }\right)$ as well as strength measures $\left(\mathrm{MM}_{\text {strength }}\right)$. Developmental disregard, bimanual performance, and capacity were assessed using a validated video-observation method. Twenty-one children with unilateral CP participated (Median age $10 \mathrm{y} 7 \mathrm{mo}$, interquartile range [IOR] 10y 1mo-12y 9mo). Outcome measures of mirror movements were correlated to developmental disregard, bimanual performance, and capacity scores using Spearman's correlations (significance level: $\alpha<0.05$ ).

RESULTS Mirror movements were not related to developmental disregard. However, enhanced mirror movements in the less-affected hand were related to reduced performance $\left(\mathrm{CCC}_{\max }\right.$ : $\left.\rho=-0.526, p=0.007 ; \mathrm{MM}_{\text {strength }}: \rho=-0.750, p<0.001\right)$ and capacity $\left(\mathrm{CCC}_{\max }: \rho=-0.410, p=0.033\right.$; $\left.\mathrm{MM}_{\text {strength }} \rho=-0.679, p<0.001\right)$. These relations were only moderate (performance: $\mathrm{MM}_{\text {strength }}$ : $\rho=-0.504, p=0.010$ ), low (capacity: $\mathrm{MM}_{\text {strength }} \rho=-0.470, p=0.016$ ) or absent for mirror movements in the affected hand. Additionally, seven children showed stronger movements in their less-affected hands when actually being asked to move their affected hand.

INTERPRETATION These findings show no relation between mirror movements and developmental disregard, but support an association between mirror movements and bimanual function.
In some children with unilateral cerebral palsy (CP), bimanual performance is more restricted than would be expected based on the capacity of the affected hand. ${ }^{1,2}$ These children appear to disregard their affected hand during typical bimanual daily activities. This non-use during spontaneous daily activities, in combination with preserved affected hand capacity, is frequently referred to as developmental disregard. ${ }^{1,3}$ Next to the direct negative impact of developmental disregard on spontaneous daily functioning, the lack of use of the affected hand might in turn also lead to reduced upper-limb function. This is because movements are not being automated and neural substrates serving entire classes of behaviours might not be established during development. ${ }^{3}$

One suggested underlying cause for developmental disregard is the persistence of mirror movements occurring in the upper-limbs. ${ }^{2,4}$ Mirror movements are simultaneous involuntary movements that accompany voluntary movements of homologous muscles on the opposite side of the body. ${ }^{4}$ For example, when one hand moves voluntarily, the other hand involuntarily performs the same action. Even though mirror movements are considered to be a normal feature of motor behaviour in young children due to immaturity of the central nervous system, they are known to gradually disappear during the first decade of life. ${ }^{5}$ However, in many children with unilateral CP these mirror movements are more pronounced and persistent. ${ }^{6}$ They are more frequently observed in the less-affected hand (LAH) when actively moving the affected hand and are reported to be stronger compared to mirror movements in the affected hand. ${ }^{4,6}$

There are two proposed mechanisms which may underlie mirror movements in children with unilateral CP. First, the motor cortex of the less-affected hemisphere is 
controlling the two hands via both contralateral projections to control the LAH, and preserved ipsilateral projections to control the affected hand movements, causing mirror movements in both, but especially in the affected hand. ${ }^{7,8}$ Second, widespread and bilateral cortical activation occurs when actively moving the affected hand related to the sensorimotor impairments of this affected hand. This lack of interhemispheric inhibition leads to motor overflow causing mirror movements in the LAH. ${ }^{7-9}$ Mirror movements in the affected hand have thus been proposed to be indicative for one motor cortex controlling both hands, ${ }^{8}$ while mirror movements in the LAH might simply be explained by sensorimotor impairments of the affected hand.

Mirror movements presented in the upper-limbs and their relation with upper-limb function has repeatedly been studied in children with unilateral CP., ${ }^{4-12}$ Even though results vary, they generally point towards an association between pronounced manual mirror movements and diminished bimanual performance. ${ }^{4,9,10}$ However, findings are inconclusive, with some studies showing associations between diminished bimanual performance and mirror movements in either hand, ${ }^{4,10}$ while others only report this association for mirror movements in the LAH. ${ }^{9}$ Still, the reported findings led authors to conclude that the symmetric nature of mirror movements hinders efficient bimanual task execution. ${ }^{4,9,10}$ Because most daily activities require asymmetrical actions of both hands (typically with the affected hand having a holding or stabilizing function), it was repeatedly suggested that pronounced mirror movements might even lead to the exclusion of the affected hand in spontaneous bimanual activities. ${ }^{2,4}$ In the typical stabilizing function of the affected hand, mirror movements in this affected hand will result in difficulties in stabilizing objects when performing manipulative tasks with the LAH. Furthermore, when actively moving the affected hand during bimanual asymmetric activities, mirror movements in the LAH cause a reduction in independent control of this 'good hand'. ${ }^{4}$ It has therefore been suggested repeatedly that mirror movements in either hand contribute to the phenomenon of developmental disregard in children with unilateral $\mathrm{CP}$ through a process of learned non-use. , $^{2,9,10}$

Although some studies have explored the relation between mirror movements and bimanual performance while controlling for the capacity of the affected hand,, 10 the relation between mirror movements and developmental disregard has never been studied directly. By using a standardized measurement to assess developmental disregard, ${ }^{13}$ the main aim of the current study was to test whether enhanced manual mirror movements are related to a greater degree of developmental disregard in children with unilateral CP. Second, by using a newly developed continuous scale with which distal manual mirror movements in both hands are registered separately (i.e. mirror movements in the affected hand when actively moving the LAH and mirror movements in the LAH when actively moving the affected hand), we aimed to clarify the relationship
What this paper adds

- Mirror movements are likely not related to developmental disregard.

- Mirror movements in the less-affected hand correlate with poor upper-limb function.

- Mirror movements in the affected hand correlate weakly with poor upperlimb function.

- In some children, mirror movements might assist affected hand movements.

between mirror movements in either hand and the previously reported impact on bimanual performance. ${ }^{4,9,10}$

\section{METHOD}

\section{Participants}

Children and adolescents with unilateral $\mathrm{CP}$, aged 7 to 18 years were recruited from different rehabilitation centres in the Netherlands and the UK. Inclusion criteria were diagnosis of unilateral CP with a Manual Ability Classification System (MACS) level of I to III. ${ }^{14}$ Many children were part of larger studies exploring neurocognitive processes, brain structures, and/or functions related to upperlimb movements using electroencephalogram (EEG), neuroimaging, and/or transcranial magnetic stimulation. The study was approved by the National Research Ethics Service (NRES), UK, as well as by the local Ethical Committee (CMO) of the region Arnhem-Nijmegen, the Netherlands. All parents provided written informed consent for participation of their children at the study as well as for publication of the results. Children over 12 also provided written assent.

\section{Clinical assessment of upper-limb capacity, performance, and developmental disregard}

For the clinical assessment of developmental disregard, upper-limb capacity, and performance, the 'revised VideoObservation Aarts and Aarts module: Determine Developmental Disregard' was used. ${ }^{13}$ Here, capacity is defined as the frequency of affected hand-use during a task that requires bimanual hand-use. Performance is defined as the frequency of affected hand-use during a task that stimulates bimanual hand-use, but is not essential to performance of the task (i.e. it is possible to perform the task with only one hand). Developmental disregard is defined as the difference between the duration of affected hand-use between both tasks, the 'demanding' and the 'stimulating' task. ${ }^{13}$ Whenever this developmental disregard score was higher than a previously validated cut-off score (i.e. 17.2\%), ${ }^{13}$ children were classified as having developmental disregard.

\section{Quantitative assessment of mirror movements}

A custom-made repetitive squeezing task was developed to quantitatively register distal manual mirror movements. During this so-called 'Windmill-task', mirror movements were assessed by placing two grip-force transducers (equipped with micro load cells: 0-5kg; weight: 45; circumference: $10 \mathrm{~cm}$ ) between thumb and index- plus middlefinger of the children's hands. When the child was not able to hold the transducer with these three fingers (e.g. due to muscle weakness or spasticity), additional fingers were 
allowed to stabilize the grip. The grip of the LAH was always matched to the grip of the affected hand: when using additional fingers with the affected hand the same fingers were used with the LAH.

One of the transducers was connected to a little windmill (Fig. 1). The motor of the windmill was programmed so the mill started rotating once the connected transducer was pressed beyond a certain threshold (approximately $1.5 \mathrm{~kg}$ ). To speed up the rotation of the mill, the grip had to be returned to a lower threshold by loosening the grip (approximately $1 \mathrm{~kg}$ ) and again reach the upper threshold within $1000 \mathrm{~ms}$, so that a repetitive squeezing pattern was induced $(\geq 1 \mathrm{~Hz}$ frequency). Children were instructed to hold the transducers in both hands with the hands lifted to

(a) Schematic of windmill and handles

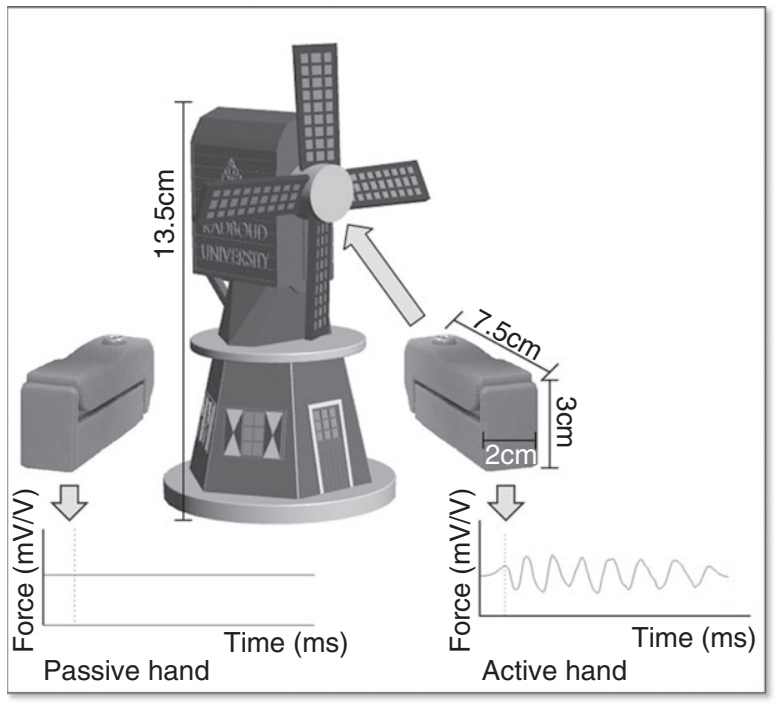

(b) Participant performing windmill task

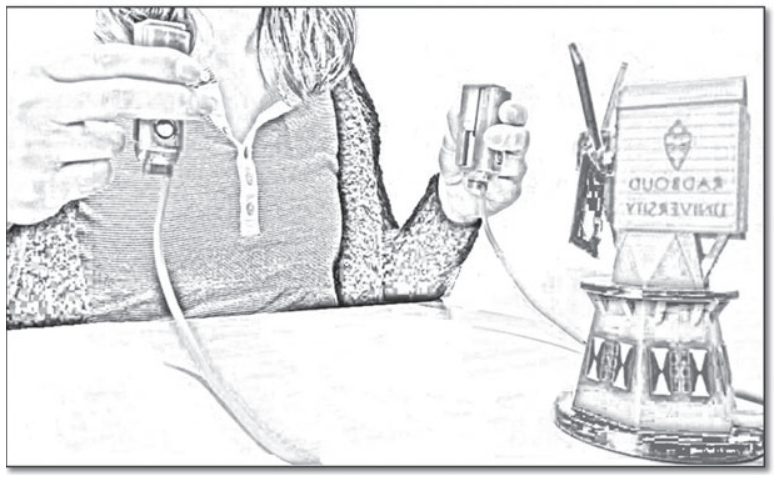

Figure 1: (a) The Windmill-task as positioned for a right-hand active squeezing. The two objects next to the windmill represent the grip-force transducers with the right transducer being connected to the windmill. Both transducers are connected to a computer, digitizing and storing the data recorded of both hands time-locked grip-force (in mV/V). This figure represents a squeezing pattern with the passive hand showing no mirror movements. (b) Participant performing the Windmill-task. chest level. With one hand (active hand) they were asked to repetitively squeeze the transducer in order to rotate the mill of the windmill as fast as possible. With the other hand, children were asked to simply lift and hold the second transducer (passive hand, tested for mirror movements). To measure the grip-force, the grip objects were equipped with strain-gauge load cells that converted the force into an electrical signal $(\mathrm{mV} / \mathrm{V})$. The time-locked grip-force signal of both hands was sampled at $50 \mathrm{~Hz}$, digitized, and stored on a computer.

\section{Procedure}

After administering the 'revised Video-Observation Aarts and Aarts module: Determine Developmental Disregard' ${ }^{13}$ participants were seated on a chair in front of a table upon which the windmill was placed. A standardized protocol of 5 seconds of squeezing and 5 seconds of rest with a total of 20 repetitions was conducted for each hand (100s of squeezing data for each hand). A pre-recorded voice indicated the start and stop for rotating the mill. Both hands were tested separately, always with the LAH first (LAH-squeezing condition) to prevent early frustration. Thus, distal manual mirror movements in both hands were tested separately: (1) mirror movements in the affected hand during LAH-squeezing; and (2) mirror movements in the LAH during affected hand-squeezing. A short practice session for each condition was conducted prior to the task (two trials of $5 \mathrm{~s}$ of squeezing).

\section{Data pre-processing}

First, to quantify mirror movements, the force pattern of both hands during each squeezing session $(20 \times 5 \mathrm{~s})$ was compared by cross-correlating both signals. ${ }^{15}$ Both gripforce signals were correlated by iteratively shifting one signal forwards in time against the other signal. A correlation-coefficient (Pearson's $r$ ) was calculated for each phase shift (steps of $20 \mathrm{~ms}$ at a $50 \mathrm{~Hz}$ sampling rate), resulting in a time series of Pearson's $r$ values. This time series was representing a correlation function at each increment of the phase shift between the two input signals. ${ }^{15}$ In a second step, an average cross-correlation function was obtained from all squeezing sessions. The maximum correlation-coefficient of this averaged function $\left(\mathrm{CCC}_{\max }\right)$ was used as an index of the similarity between the two squeezing signals. Hence, $\mathrm{CCC}_{\max }$ is indicative of the intensity of mirror movements, with $r=0$ reflecting no mirroring of the passive hand during active hand movement, and $r=1$ reflecting that the passive hand is performing the exact same movement as the active hand. Whenever $\mathrm{CCC}_{\max }$ was $\geq 0.30$, children were classified as having mirror movements, as a correlation-coefficient $<0.30$ is considered negligible. ${ }^{16}$

To further operationalize the intensity of the mirror movements, the mean grip-force of the passive hand during each squeezing session was calculated as the difference between the peaks and the troughs of the force signal. These values were averaged across all trials and normalized 
by dividing them by the mean force values of the same hand when actively squeezing $\left(M_{\text {strength }}\right)$. A higher $M M_{\text {strength }}$ indicated increased strength in the passive hand during the squeezing period, hence pronounced mirror movements.

$\mathrm{CCC}_{\max }$ and $\mathrm{MM}_{\text {strength }}$ calculations were performed separately for both conditions (affected hand-squeezing vs. LAH-squeezing). The active squeezing period started $500 \mathrm{~ms}$ after the 'start' signal and lasted 5 seconds to control for the slight delay following the auditory 'start' signal. All trials were individually inspected and excluded from the analyses if the active hand did not show a repetitive squeezing pattern (at least five repetitions $\geq 1 \mathrm{~Hz}$ ) within this period $(3.1 \%$ data exclusion in the affected handsqueezing condition; $1.2 \%$ in the LAH-squeezing condition).

\section{Statistical analysis}

Shapiro-Wilk tests indicated that most variables were not normally distributed (only the $\mathrm{CCC}_{\max }$ variables for both conditions were normally distributed). Furthermore, only small numbers of participants $(n<30)$ were included for the current study. Therefore, for statistical analysis non-parametric tests were applied.

Aim 1: To test the relation between enhanced distal hand mirror movements and higher developmental disregard scores, $\mathrm{CCC}_{\max }$ and $\mathrm{MM}_{\text {strength }}$ values were related to the individuals' developmental disregard scores for both conditions separately (affected hand-squeezing vs. LAHsqueezing) using one-tailed Spearman rank $(\rho)$ correlations.

Aim 2: To clarify the relation between mirror movements in either hand and upper-limb function, we first verified whether mirror movements were stronger in the LAH when the affected hand was actively moving. Two Wilcoxon Signed-Rank tests were used to compare $\mathrm{CCC}_{\max }$ and $\mathrm{MM}_{\text {strength }}$ scores between both hands.

Subsequently, one-tailed Spearman rank $(\rho)$ correlations were applied between 'revised Video-Observation Aarts and Aarts module: Determine Developmental Disregard ${ }^{13}$ outcomes of upper-limb performance and $\mathrm{CCC}_{\max }$ and $M M_{\text {strength }}$ scores for both conditions separately (affected hand-squeezing vs. LAH-squeezing). The same was done for the 'revised Video-Observation Aarts and Aarts module: Determine Developmental Disregard ${ }^{13}$ upper-limb capacity scores.

For all analyses, the significance level was set at $\alpha<0.05$. Correlation coefficients $>0.70$ were considered as high, 0.50 to 0.70 as moderate, 0.50 to 0.30 as low, and $<0.30$ as negligible. ${ }^{16}$

\section{RESULTS}

\section{Participants}

Twenty-three children and adolescents with unilateral CP participated. Two were excluded as they were not able to perform the task with their affected hand (MACS III). For the remaining 21 participants (12 males; 5 MACS I, 14 MACS II, 2 MACS III) the median age was 10 years and
7 months (IQR 10y 1mo-12y 9mo; 12 affected hand right). Nine participants were classified as having developmental disregard (developmental disregard score $\geq 17.2 \%$; six males; 6 affected hand right; $\bar{x}_{\text {age }}=10 \mathrm{y} 8 \mathrm{mo}$ ). Seven children did not show any mirror movements $\left(\mathrm{CCC}_{\max }<0.30\right.$; four males; 4 affected hand right; $\bar{x}_{\mathrm{age}}=11 \mathrm{y}$ $1 \mathrm{mo})$; six children showed mirror movements only in the LAH when the affected hand was actively moving (four male; 4 affected hand right; $\bar{x}_{\text {age }}=10 \mathrm{y} 6 \mathrm{mo}$ ); seven children showed mirror movements in both hands (four male; 4 affected hand right; $\bar{x}_{\mathrm{age}}=12 \mathrm{y} 10 \mathrm{mo}$ ), and one child showed only mirror movements in the affected hand when the LAH was actively moving (male; affected hand right; age $=7 \mathrm{y} 1 \mathrm{mo})$.

Aim 1: Developmental disregard scores were not related to mirror movements in the affected hand $\left(\mathrm{CCC}_{\max }\right.$ : $\left.\rho=0.091, p=0.348 ; M_{\text {strength }} \rho=0.201, p=0.191\right)$ or $\mathrm{LAH}$ $\left(\mathrm{CCC}_{\max }: \quad \rho=-0.113, \quad p=0.313 ; \quad \mathrm{MM}_{\text {strength }}: \rho=0.129\right.$, $p=0.289$ ).

Aim 2: More mirror movements were observed in the LAH, evidenced by significantly higher $\mathrm{CCC}_{\max }$ and $\mathrm{MM}_{\text {strength }}$ values when the affected hand was actively moving $\left(\mathrm{CCC}_{\max }\right.$ : Median=0.39, IQR 0.20-0.67; $\mathrm{MM}_{\text {strength }}$ : Median $=0.077$, IQR 0.009-0.792) compared with when the LAH was actively moving $\left(\mathrm{CCC}_{\max }:\right.$ Median $=0.22, \mathrm{IQR}$ 0.13-0.49, $\quad p=0.046 ; \quad M_{\text {strength: }}$ Median $=0.065$, $\mathrm{IQR}=0.019-0.144, p=0.035$; see Fig. 2).

For mirror movements in the LAH, correlation analyses showed moderate to high associations between low scores on upper-limb performance and enhanced mirror movements. This was evidenced by a significant negative correlation between performance and $\mathrm{CCC}_{\max }$ scores ( $\rho=-0.526, p=0.007)$ as well as between performance and $\mathrm{MM}_{\text {strength }}$ values $(\rho=-0.750, p<0.001)$. For upper-limb capacity, low to moderate significant negative correlations were observed $\left(\mathrm{CCC}_{\max }: \rho=-0.410, p=0.033 ; \mathrm{MM}_{\text {strength }}\right.$ : $\rho=-0.679, p<0.001$; see Fig. 3, 'Affected hand-squeezing').

Mirror movements in the affected hand also showed significant, low to moderate negative correlations with bimanual performance $\left(M_{\text {strength }}: \rho=-0.504, p=0.010\right)$ and capacity scores $\left(M M_{\text {strength }}: \rho=-0.470, p=0.016\right.$; see Fig. 3, 'LAH squeezing'). Correlations between $\mathrm{CCC}_{\max }$ scores and bimanual performance $(\rho=-0.352, p=0.059)$ as well as capacity $(\rho=-0.191, p=0.204)$ did not reach significance.

\section{Additional findings}

During the 'affected hand-squeezing' condition seven children (five male, 3 affected hand right, Median age: $11 \mathrm{y} 10 \mathrm{mo}$ ) displayed a stronger force pattern in the passive, LAH (Median 2831.35, IQR 2275.01-2924.79) compared with the active, affected hand (Median 1655.80, IQR 1065.66-1949.10). In all of these seven children, the time-lag information of the $\mathrm{CCC}_{\max }$ values were negative, indicating that the LAH was moving slightly before the affected hand. This pattern was only observed in children with greater impairments in manual ability $(\mathrm{MACS} \geq 2)$. 
(a)

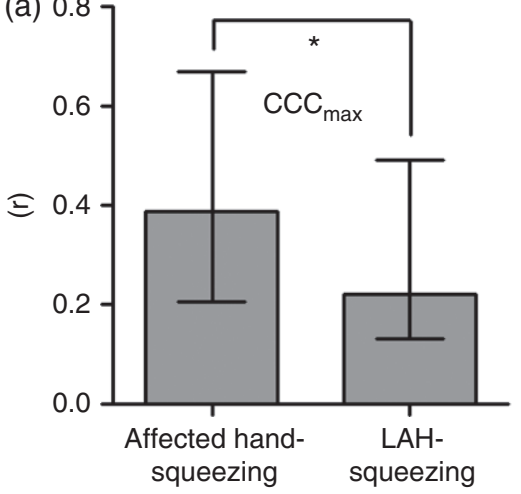

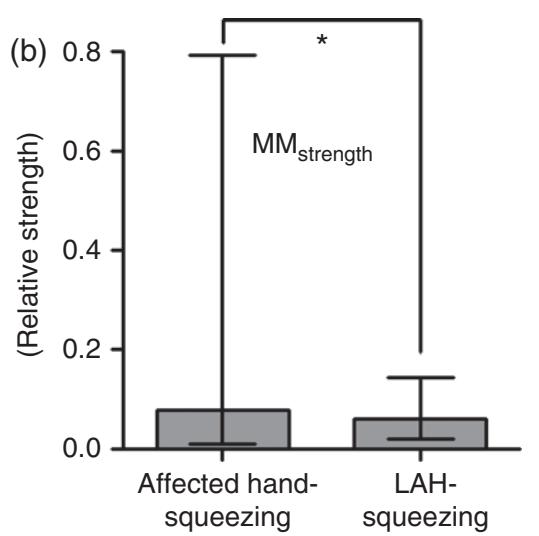

Figure 2: $\mathrm{CCC}_{\max }(\mathrm{a})$ and $\mathrm{MM}_{\text {strength }}(\mathrm{b})$ values representing the intensity of mirror movements per condition (left: affected hand actively squeezing; right: LAH actively squeezing) represented by the medians and interquartile ranges. $\mathrm{CCC}_{\max }$ values indicate the averaged maximum cross-correlation between both hands force signals, with higher values reflecting more similarity between both force patterns, hence more mirror movements. $\mathrm{MM}_{\text {strength }}$ values indicate the strength of the passive hand during the active squeezing period, with higher values indicating stronger mirror movements. $\mathrm{LAH}$, less-affected hand.

\section{DISCUSSION}

The main finding of the current study is that distal manual mirror movements during a unimanual squeezing task in unilateral CP are not related to the phenomenon of developmental disregard. Earlier studies have suggested a direct relation between manual mirror movements and non-use or disregard of the affected hand. ${ }^{2,9,10}$ This suggestion was based on the observed association between pronounced mirror movements and diminished bimanual performance., ${ }^{4,9,10}$ It has been argued that when mirror movements occur in the affected hand, which mostly has a holding or stabilizing function, mirror movements cause less stability; furthermore, mirror movements cause a reduction in independent control of the LAH when occurring while actively moving the affected hand during bimanual asymmetric activities. ${ }^{4}$ Our findings concur with these hypotheses, showing a relation between pronounced mirror movements in either hand and diminished bimanual performance. Previous hypotheses have posited that mirror movements may therefore lead to a non-use of the affected hand in spontaneous bimanual activities, i.e. developmental disregard. ${ }^{9,10}$ The present study is the first to directly test this suggested relation between manual mirror movements and developmental disregard, and results show a lack of this association.

The factors contributing to the phenomenon of developmental disregard are not yet fully understood. Originally, it was argued that developmental disregard is a behavioural phenomenon, resulting from the negative experience each time the affected hand is used. ${ }^{17}$ However, recent experimental findings aimed at unravelling developmental disregard, ${ }^{18,19}$ as well as related theoretical frameworks, ${ }^{2,3,20,21}$ suggest that this phenomenon may also be the result of compromised visuo-spatial attention as well as a developmental delay related to higher order motor executive functions, thereby challenging the earlier accounts of developmental disregard. ${ }^{16}$ Our current finding - that mirror movements are not related to developmental disregard - adds to this body of knowledge by showing that reduced bimanual efficiency does not necessarily lead to developmental disregard in children with unilateral CP.

Another important facet of our study was the clarification of the nature of the relationship between distal manual mirror movements and bimanual performance. This was done by using an objective quantitative assessment tool to assess distal hand mirror movements in both hands separately and relating this data to a bimanual performance measure. Previously reported results have been inconclusive, with some study results showing associations between diminished bimanual performance and mirror movements in both hands, ${ }^{4,10}$ while other findings only report this association for mirror movements appearing in the LAH. ${ }^{9}$ However, these earlier studies either used only a subjective, ordinal rating scale for assessing mirror movements ${ }^{9,10}$ or lacked standardized testing when assessing bimanual performance. ${ }^{4}$ We were able to show significant moderate to high correlations between bimanual performance and mirror movements appearing in the LAH when the affected hand was actively moving. Additionally, low to moderate correlations were also observed between bimanual performance and mirror movements appearing in the affected hand when the LAH was actively moving. By confirming these relations, we showed that mirror movements in either hand might be related to reduced performance during bimanual asymmetric activities. At the same time, our finding of a lack of relation between these mirror movements and developmental disregard indicates that this does not necessarily lead to a non-use or disregard of the affected hand during spontaneous daily activities.

Next to the explanation that mirror movements are directly related to a reduced performance during bimanual 
(a) Maximum cross-correlation coefficient $\left(\mathrm{CCC}_{\max }\right)$
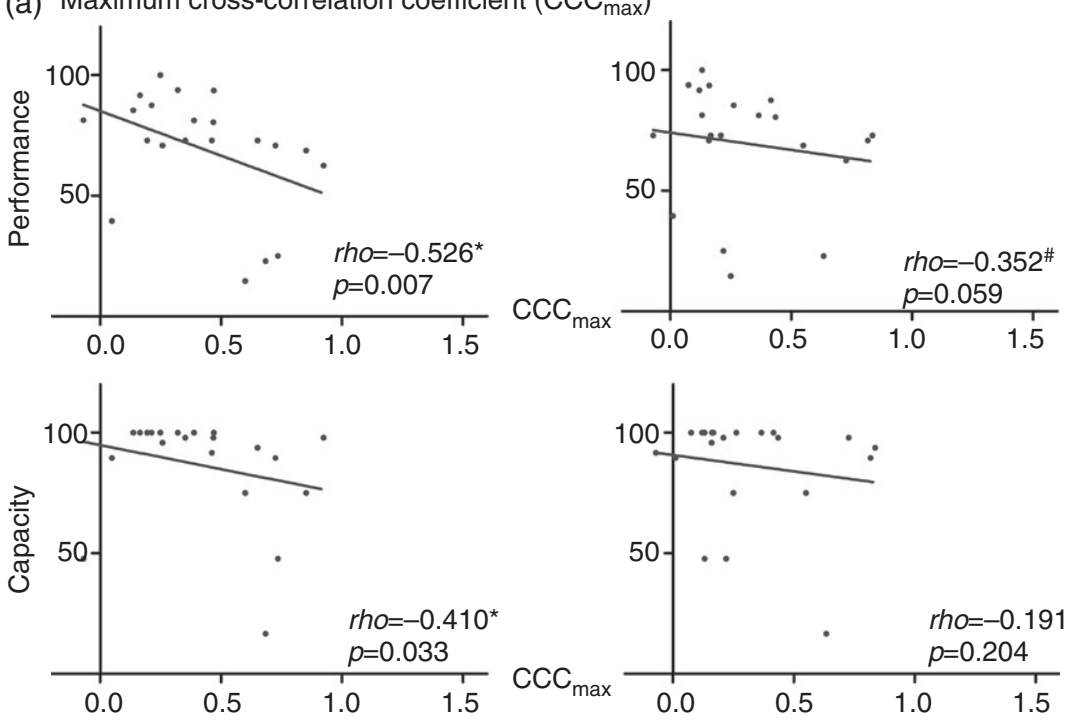

(b) Strength of passive hand ( $\left.\mathrm{MM}_{\text {strength }}\right)$
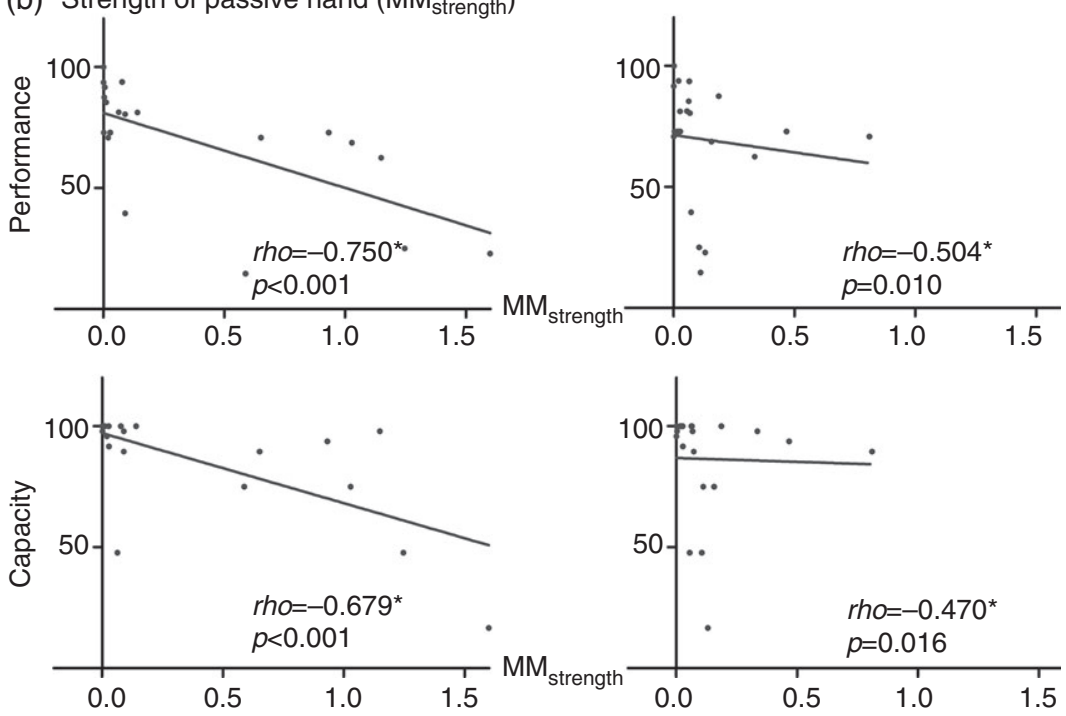

Figure 3: Depicted are correlations between upper-limb function (performance and capacity) and mirror movements, with * representing significance $(p<0.05)$ and ${ }^{\#}$ representing a statistical trend $(p<0.1$ and $>0.05)$. (a) Correlations between upper-limb function (upper graphs: performance; lower graphs: capacity) and the cross-correlation between both hands force signals $\left(\mathrm{CCC}_{\max }\right)$. (b) Correlation between upper-limb function (upper graphs: performance; lower graphs: capacity) and the strength of the passive hand during the active squeezing condition $\left(\mathrm{MM}_{\text {strength }}\right)$. LAH, less-affected hand.

asymmetric activities, the negative correlation between bimanual performance and mirror movements might also simply be explained by the type and/or severity of the children's lesion. The neuropathology would then in turn explain both, the reduced bimanual performance as well as the enhanced mirror movements (due to widespread bilateral activation during unimanual movements or even ipsilateral cortico-spinal connections from the less-affected hemisphere to the affected hand). ${ }^{7-9}$ This interpretation is supported by the current finding that mirror movements were also correlated to hand capacity, as has been reported previously. ${ }^{9}$ However, without details of the extent and location of the individual lesions or direct unimanual capacity measures, it is not possible to elaborate on the cause of the observed reduction in bimanual performance.

Our results furthermore replicated earlier findings that many children with unilateral CP display significantly more mirror movements in the LAH compared with the affected hand. ${ }^{4,6,9}$ There are three potential explanations for this finding. First, the more dextrous use of the LAH compared 
with the affected hand might contribute to a more discrete and lateralized pattern of neural control of the LAH compared with the affected hand, leading to reduced mirror movements in the affected hand when the LAH is actively moving. ${ }^{4,9}$ Second, the enhanced mirror movements in the LAH might be related to the sensorimotor impairments of the affected hand, and evolve due to inefficient interhemispheric inhibition from the affected hemisphere, resulting in bilateral excitatory activity. ${ }^{19}$ Third, mirror movements appearing in the LAH might represent a non-specific motor overflow phenomenon which indirectly assists affected hand movements. ${ }^{9}$ This latter explanation is based on the notion that children with reduced manual ability of the affected hand may move both hands simultaneously when asked to only move their affected hand, in order to overcome the lack of selectivity and strength of their affected hand. This is because symmetrical movements are performed more easily. ${ }^{22}$ This possible assisting strategy might be especially useful during predominantly symmetric bimanual activities and potentially also during the less frequently observed phenomenon of unimanual affected hand movements (e.g. releasing an object by actively opening the LAH). Thus, mirror movements in the LAH may in some cases be considered to assist controlled movements of the affected hand.

In line with the suggestion that mirror movements appearing in the LAH might occur to assist affected hand movement, we found that seven children displayed a stronger force pattern in the LAH when they were asked to actively move their affected hand. These children also started moving their LAH slightly earlier. This additional finding may imply that these children facilitate the movement of their affected hand by moving their LAH. That is, they appear to 'use' their mirror movements as a strategy to facilitate movements of the affected hand. This pattern was only observed in children with reduced manual ability (MACS $\geq 2)$. Therefore, the slight delay of the affected hand movement might also be explained by biomechanical processes related to this reduced manual ability. Further research is warranted to unravel the possible strategic use in particular, to answer the question of whether this possible strategic use of mirror movements leads to a better unimanual or bimanual control of the affected hand during some daily activities.

The current study was limited by the small group size, especially of the more severely impaired children (i.e. MACS III). Additionally, two children had to be excluded as they were not able to complete the task with their affected hand. For future studies, the task needs to be adapted in a way that the thresholds for moving the windmill are scaled to the individuals' maximal force capacities. Another limitation affecting performance is our block design, where the LAH always started. This may have led to possible carry-over effects that would have been avoided with a randomized design. Finally, and inherent to the studied population, is the heterogeneity of the studied group (e.g. aetiology, underlying differences in brain injury).

To conclude, no relation between mirror movements and developmental disregard in children with unilateral CP was observed. Using a newly developed quantitative tool to assess mirror movements, earlier findings on mirror movements were supported: mirror movements were related to reduced manual performance. Furthermore, mirror movements were shown to be stronger in the LAH during affected hand movement. Finally, in a subset of the children, our new quantitive measurement uncovered a possible strategy to use mirror movements to control movements of the affected hand.

\section{ACKN OWLEDGEMENTS}

We want to thank all the children and their families who took part in this study. We also want to kindly acknowledge Mark van de Hei and Pascal de Water from the technical support group of the Radboud University Nijmegen for their valuable and professional assistance in developing the 'Windmill-task'. Furthermore, we kindly thank Sabine Vincon for her help in collecting the clinical data. This work is part of a doctoral dissertation that was supported by grants from (in alphabetical order): Hersenstichting Nederland, Johanna KinderFonds, Stichting Rotterdams Kinderrevalidatie Fonds Adriaanstichting, Phelps Stichting voor Spastici, and Revalidatiefonds. The authors have stated that they had no interests which might be perceived as posing a conflict or bias.

\section{REFERENCES}

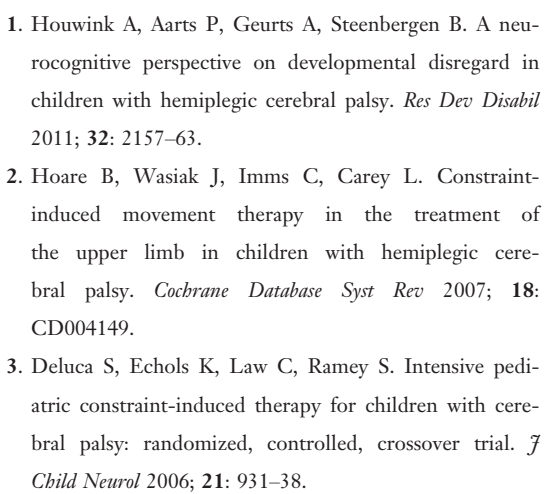

8. Jaspers E, Byblow WD, Feys H, Wenderoth N. The corticospinal tract: a biomarker to categorize upper limb functional potential in unilateral cerebral palsy. Fron Pediatr 2016; 3: 112.

9. Klingels K, Jaspers E, Staudt M, Guzzetta A, Mailleux L, Ortibus E, Feys H. Do mirror movements relate to hand function and timing of the brain lesion in children with unilateral cerebral palsy? Dev Med Child Neurol 2016; 58: $735-42$.

10. Adler C, Berweck S, Lidzba K, Becher T, Staudt M. Mirror movements in unilateral spastic cerebral palsy: specific negative impact on bimanual activities of daily living. Eur 7 Paediatr Neurol 2015; 19: 504-09. 
11. Islam M, Gordon AM, Skold A, Forssberg H, Eliasson AC. Grip force coordination during bimanual tasks in unilateral cerebral palsy. Dev Med Child Neurol 2011; 53: 920-26.

12. Holmstrom L, Vollmer B, Tedroff K, et al. Hand function in relation to brain lesions and corticomotor-projection pattern in children with unilateral cerebral palsy. Dev Med Child Neurol 2010; 52: 145-52.

13. Houwink A, Geerdink Y, Steenbergen B, Geurts A, Aarts P. Assessment of upper-limb capacity, performance, and developmental disregard in children with cerebral palsy: validity and reliability of the revised Video-Observation Aarts and Aarts module: Determine Developmental Disregard (VOAA-DDD-R). Dev Med Child Neurol 2013; 55: 76-82.

14. Eliasson A-C, Krumlinde-Sundholm L, Rösblad B, et al. The Manual Ability Classification System (MACS) for children with cerebral palsy: scale development and evi- dence of validity and reliability. Dev Med Child Neurol 2006; 48: 549-54.

15. Nelson-Wong E, Howarth S, Winter DA, Callaghan JP. Application of autocorrelation and cross-correlation analyses in human movement and rehabilitation research. 7 Orthop Sports Phys Ther 2009; 39: 287-95.

16. Mukaka MM. Statistics corner: a guide to appropriate use of correlation coefficient in medical research. Malawi Med 7 2012; 24: 69-71.

17. Taub E, Uswatte G, Mark V, Morris DM. The learned nonuse phenomenon: implications for rehabilitation. Eura Medicophys 2006; 42: 241-56.

18. Zielinski IM, Jongsma ML, Baas CM, Aarts PB, Steenbergen B. Unravelling developmental disregard in children with unilateral cerebral palsy by measuring event related potentials during a simple and complex task. BMC Neurol 2014; 14: 6
19. Zielinski IM, Steenbergen B, Baas C, Aarts P, Jongsma M. Neglect-like characteristics of developmental disregard in children with cerebral palsy revealed by event related potentials. BMC Neurol 2014; 14: 221.

20. Sutcliffe T, Logan W, Fehlings D. Pediatric constraint-induced movement therapy is associated with increased contralateral cortical activity on functional magnetic resonance imaging. 7 Child Neurol 2009; 24 : 1230-35.

21. Weinstein M, Green D, Geva R, et al. Interhemispheric and intrahemispheric connectivity and manual skills in children with unilateral cerebral palsy. Brain Struct Funct 2014; 219: 1025-40.

22. Swinnen SP, Wenderoth N. Two hands, one brain: $\operatorname{cog}$ nitive neuroscience of bimanual skill. Trends Cogn Sci 2004; 8: 18-25.

\section{New from Mac Keith Press Practical Guide}

\section{Developmental Assessment}

Theory, Practice and Application to Neurodisability

\section{Patricia M Sonksen}

- Clarifies 'what is being tested' and explains the rationale behind traditional assessment tests.

- Includes tables of age guidelines that provide quick access to the ages when $50 \%$ and $90 \%$ of children achieve a specific domain.

- Describes permissible adaptations for children with impairments as well as the design of intervention strategies.

Contact us at admin@mackeith.co.uk to receive further details.

March 2016 / 240 x 170mm / 384 pp / Paperback / 978-1-909962-56-9 / £39.95

www.mackeith.co.uk/shop 